\title{
ARTÍCULO ORIGINAL \\ Afrontamiento y adaptación en cuidadores principales de niños hospitalizados, según el modelo de Callista Roy
}

\author{
Coping and adaptation in primary caregivers of hospitalized \\ children, based on Callista Roy's model / Enfrentamento e \\ adaptação em cuidadores primários de crianças hospitalizadas, \\ segundo o modelo Callista Roy
}

Dora Isabel Giraldo Montoya ${ }^{1}$, Libia María Rodríguez Padilla², María del Pilar Vargas Betancur ${ }^{3}$, Mariana Suárez Yepes ${ }^{3}$, Lucy Neira Trujillo Bedoya ${ }^{4}$, Carolina Rodríguez Obando5, Salomé Cardona López ${ }^{5}$

\section{RESUMEN}

Objetivo: identificar el nivel de afrontamiento y adaptación de los cuidadores principales de niños hospitalizados en dos centros de Medellín, desde el modelo Callista Roy, durante el año 2019.

Metodología: estudio transversal descriptivo prospectivo. El tamaño de muestra fue de 400 cuidadores principales. Se incluyeron mayores de 18 años, quienes firmaron el consentimiento informado. Se utilizó una encuesta sociodemográfica y la Escala de Medición del Proceso de Afrontamiento y Adaptación, versión en español (EsCAPS, abreviada), previa autorización. Las variables cualitativas se presentan en frecuencias absolutas y relativas, y las cuantitativas en mediana acompañada del rango intercuartílico (RIC).

Resultados: todos los cuidadores eran mujeres, con mediana de edad de 30 años y RIC 25-37, 119 (29,5\%) tenían un nivel educativo técnico y tecnólogo, su estrato socioeconómico estaba entre los estratos uno, dos y tres (84.6\%). De ellas, 160 (39.7\%) vivían en unión libre. En cuanto a los niños, la mediana de edad fue de 24 meses (RIC 2-72). El diagnóstico principal fue de afecciones respiratorias, 113/398 (28.4\%). La mediana del puntaje de afrontamiento global fue 56 (RIC 50-60), lo que ubica a la mayoría en un nivel de afrontamiento bajo.

Conclusión: los cuidadores principales de niños hospitalizados tienen un grado bajo de afrontamiento, es necesario fortalecer los planes de cuidado para mejorar el bienestar de los cuidadores.

Palabras clave: adaptación psicológica; cuidadores; pediatría; hospitalización; enfermeros.

\section{ABSTRACT}

Objective: To identify the level of coping and adaptation of primary caregivers of hospitalized children during 2019 in two centers of Medellin, based on Callista Roy's model. Methodology: Prospective descriptive cross-sectional study. The sample size was made up of 400 primary caregivers. Participants over 18 years who signed the informed consent were included. A sociodemographic survey and the Coping Adaptation Processing Scale version in Spanish (abbreviated EsCAPS) were used, with prior authorization. Qualitative variables were expressed in absolute and relative frequencies, while quantitative ones were expressed using median and interquartile range (IQR).

Results: All the caregivers were women, with a median age of 30 years and IQR: 25-37. 119 (29.5\%) were technicians and technologists, who belonged to socioeconomic strata 1,2 , and 3 (84.6\%). 160 (39.7\%) of them lived in a common-law marriage. Amongst hos-
Fecha de recibido:

21 de noviembre de 2020 .

Fecha de aprobación: 2 de marzo de 2021.

Forma de citar este artículo: Giraldo DI, Rodríguez LM, Vargas MP, Suárez M, Trujillo LN, Rodríguez $C$, et al. Afrontamiento y adaptación en cuidadores principales de niños hospitalizados, según el modelo de Callista Roy. Med UPB. 2021;40(2):13-21. DOI:10.18566/medupb.v40n2.a03

\footnotetext{
1 Facultad de Enfermería, Escuela de Ciencias de la Salud, Universidad Pontificia Bolivariana. Medellín, Colombia.

2 Facultad de Medicina, Escuela de Ciencias de la Salud, Universidad Pontificia Bolivariana. Medellín, Colombia.

3 Servicio de Pediatría, Hospital Pablo Tobón Uribe. Medellín, Colombia.

4 Servicio de Pediatría, Clínica Universitaria Bolivariana. Medellín, Colombia.

${ }^{5}$ Semillero de Investigación Cuidar. Facultad de Enfermería, Universidad Pontificia Bolivariana. Medellín, Colombia.
}

\section{Dirección de}

correspondencia: Dora Isabel Giraldo Montoya. Correo electrónico: dora. giraldo@upb.edu.co 
pitalized children, the median age was 24 months and the IQR 2-72; the most frequent diagnosis was respiratory disorders, $113 / 398$ (28.4\%). The median global score was 56 , with an IQR of 50-60, placing most of them in a low coping level.

Conclusion: Primary caregivers of hospitalized children have a low level of coping. It is necessary to strengthen the care plans for the welfare of caregivers.

Keywords: psychological adaptation; caregivers; pediatrics; hospitalization; nurses

\section{RESUMO}

Objetivo: identificar o nível de enfrentamento e adaptação dos cuidadores principais de crianças hospitalizadas em dois centros de Medellín, a partir do modelo Callista Roy, durante 2019.

Metodologia: estudo transversal descritivo prospectivo. O tamanho da amostra foi de 400 cuidadores principais. Foram incluídos os maiores de 18 anos que assinaram o Termo de Consentimento Livre e Esclarecido. Utilizou-se um levantamento sociodemográfico e a Escala de Medição do Processo de Enfrentamento e Adaptação, versão em espanhol (EsCAPS, abreviatura), com autorização prévia. As variáveis qualitativas são apresentadas em frequências absolutas e relativas e as variáveis quantitativas em mediana acompanhadas do intervalo interquartil (IQR).

Resultados: todos os cuidadores eram mulheres, com mediana de idade de 30 anos e IQR 25-37, 119 (29,5\%) possuíam escolaridade técnica e tecnológica, estrato socioeconômico entre os estratos um, dois e três (84,6\%). Destes, 160 (39,7\%) viviam em união estável. Em relação às crianças, a mediana de idade foi de 24 meses (IQR 2-72). $O$ diagnóstico principal foram doenças respiratórias, 113/398 (28,4\%). A pontuação geral mediana de enfrentamento foi de 56 (IQR 50-60), o que coloca a maioria em um nível de enfrentamento baixo.

Conclusão: os cuidadores principais de crianças hospitalizadas apresentam baixo grau de enfrentamento, é necessário fortalecer os planos de cuidado para melhorar o bemestar dos cuidadores.

Palavras-chave: adaptação psicológica; cuidadores; pediatria; hospitalização; enfermeiras

\section{INTRODUCCIÓN}

Según Gutiérrez López, el afrontamiento y la adaptación son muy importantes para la calidad de vida, dado que son procesos dinámicos de integración entre el individuo y su entorno, que generan estilos y estrategias para resolver una situación ${ }^{1}$. El modelo de Callista Roy, analizado por profesores de la Universidad de la Sabana ${ }^{2}$, plantea que el profesional de enfermería, al establecer una relación con el paciente, debe tener en cuenta la capacidad de autodeterminación de la persona y los mecanismos que emplea para adaptarse.

Con el propósito de identificar de qué modo la persona utiliza sus habilidades para controlar situaciones que ocasionan estrés y promover su adaptación, Callista Roy diseñó en el año 2004 la escala Coping Adaptation Processing Scale (CAPS). La CAPS tiene 47 ítems que se agrupan en cinco factores ${ }^{3}$. Posteriormente, un grupo de profesoras de la Universidad de la Sabana desarrolló una versión modificada de la escala para el ámbito colombiano, compuesta por 33 ítems. La escala reportó validez de expertos del 0.95 y de contenido de $0.83^{4}$.
En pediatría varios estudios han usado la CAPS para identificar el grado de afrontamiento y de adaptación de los padres o cuidadores principales de niños con problemas de salud. Entre estos trabajos es de mencionar el de Campiño et $\mathrm{al}^{5}$, quienes encontraron que el grado de afrontamiento de los cuidadores de niños con cáncer fue alto, así como el de Suárez y Monroy ${ }^{6}$, que también reporta un puntaje alto. De otro lado, la investigación de Enríquez et a $1^{7}$ encontró un puntaje medio, lo mismo que la de Ochoa et at ${ }^{8}$.

Tobo et $\mathrm{al}^{9}$ refieren que la hospitalización produce en los padres sentimientos negativos y estrés, por lo que es primordial apoyar y fomentar su participación en la atención, a fin de aumentar su confianza y grado de afrontamiento en relación con el cuidado de su hijo. Así mismo, es necesario evaluar constantemente los factores estresantes para los padres. En este sentido, la enfermería tiene un papel clave en el proceso de adaptación de los pacientes y su familia, en especial los padres o cuidadores principales, como responsables directos del cuidado. Los cuidadores principales tienen que resolver necesidades físicas, económicas, sociales, culturales y emocionales, tanto en el hospital como fuera de él, que exigen reor- 
ganizar la vida familiar, laboral y social en función de las tareas que implica cuidar ${ }^{10}$. Las estrategias y los estilos de afrontamiento que utilicen los cuidadores hacen parte de los determinantes del curso de la enfermedad de niños ${ }^{11}$.

El objetivo de este estudio es identificar el grado de afrontamiento y adaptación de los cuidadores principales de niños hospitalizados en pediatría, según el modelo de Callista Roy, a través de la versión abreviada de la escala CAPS en español (EsCAPS abreviada).

\section{METODOLOGÍA}

\section{Diseño, población de estudio y criterios de inclusión}

Estudio transversal descriptivo de las cuidadoras de niños hospitalizados en la Clínica Universitaria Bolivariana (CUB) y en el Hospital Pablo Tobón Uribe (HPTU), ambas instituciones de salud de tercer nivel de complejidad de la ciudad de Medellín, Colombia, durante un semestre de 2019.

Se incluyeron mayores de 18 años que fueran madres biológicas o cuidadoras principales (madres sustitutas y abuelas) de los niños hospitalizados en los servicios de pediatría de ambas instituciones.

\section{Estimación de tamaño de la muestra}

Se estimó una muestra de cuidadoras según el total esperado en las dos instituciones (1 188), según la premisa de que mensualmente ingresan en promedio 60 niños a hospitalización en la CUB y 138 al HPTU, para un total de atención por semestre de 360 y 828 , respectivamente. A partir de esta población total esperada se calculó un tamaño de muestra mínimo de 400 niños, con sus respectivos cuidadores principales, que era necesario para cumplir con los objetivos del estudio. Para este cálculo se tuvo en cuenta una proporción esperada de afrontamiento de grado bajo de $14.8 \%$, un nivel de confianza del $95 \%$, una precisión del $4 \%$, un efecto de diseño de 1.5 y un sobremuestreo del $10 \%$. También, se planeó encuestar a 124 cuidadores en la CUB y 276 del HPTU, para garantizar que en la muestra quedara una representación de cada institución, proporcional al tamaño de la población esperada en un semestre.

\section{Proceso de recolección de la información}

Una vez se obtuvo el aval ético y administrativo se inició el proceso de recolección de datos. Para ello, semanalmente los investigadores contactaron a los cuidadores de los niños hospitalizados que cumplían los criterios de selección, les socializaron los objetivos del estudio y les solicitaron, mediante consentimiento informado, su participación. La muestra mínima se captó de manera consecutiva, en la medida en que ingresaban los niños al servicio de hospitalización en cada institución, hasta completar el número de cuidadores necesarios.

Cada participante diligenció una encuesta que contenía aspectos sociodemográficos, tanto del cuidador como del niño (edad del cuidador, tipo de cuidador, ocupación, grado de escolaridad, estrato socioeconómico, estado civil, religión, edad del niño), características clínicas o del cuidado del niño (diagnóstico principal, tiempo de cuidado, apoyo en el cuidado del niño, entre otras) y la EsCAPS abreviada (usada con la autorización correspondiente). La EsCAPS fue validada en la población colombiana en el año 2013, una validez de expertos (aparente) de 0.95, un índice de validez de contenido según propuesta de Lawshe de 0.83 y una consistencia interna según el alfa de Cronbach de $0.711^{14}$. Las autoras de la escala EsCAPS abreviada refieren que, el análisis factorial de la versión de 33 ítems no mantuvo la estructura de los cinco factores de la versión original, razón por la cual el presente estudio no midió los resultados para estos.

La EsCAPS abreviada permitió identificar el grado de afrontamiento y adaptación de los cuidadores de niños hospitalizados, mediante la utilización de los 33 ítems en formato Likert, con cuatro opciones de respuesta (nunca: 0 puntos, casi nunca: 1 punto, casi siempre: 2 puntos y siempre: 3 puntos), cuyas puntuaciones oscilaron entre 0 y 3. Cada ítem evalúa, mediante una frase, cómo una persona responde cuando experimenta una crisis o un evento difícil y el puntaje global de la escala se consigue de la sumatoria de las puntuaciones obtenidas en cada ítem, lo que permite una calificación mínima de cero y una máxima de 99 puntos. Según la puntuación total obtenida por cada participante, el grado de afrontamiento se clasificó en bajo (0-56 puntos), medio (57-70 puntos) y alto (71-99 puntos). A continuación, en la Tabla 1 se reportan los ítems evaluados.

\section{Análisis estadístico}

Las variables cualitativas se expresaron en frecuencias absolutas y relativas, mientras que las cuantitativas en mediana, acompañada del rango intercuartílico (RIC), dado el no cumplimiento del supuesto de normalidad de los datos, verificado mediante la prueba de KolmogórovSmirnov $(\mathrm{p}<0.05)$. Todos los análisis se realizaron en el programa IBM SPSS 25.

\section{Consideraciones éticas}

Esta investigación se consideró de riesgo mínimo según la resolución 8430 de 1993, vigente en la legislación colombiana, y fue aprobado por el Comité de Investigación en Salud de la UPB, mediante acta número 10 de 2018. Además, contó con el aval de las dos instituciones hos- 
Tabla 1. Ítems de la escala EsCAPS abreviada.

1. Cuando tengo un problema o situación difícil, puedo seguir varias indicaciones al mismo tiempo

2. Cuando tengo un problema inesperado encuentro soluciones nuevas para resolverlo

3. Cuando tengo un problema o situación difícil acepto el problema como es o como se presenta

4. Cuando estoy preocupado por un problema o situación difícil tengo dificultad para completar actividades, tareas y proyectos

5. Cuando tengo un problema o situación difícil solo puedo pensar en lo que me preocupa del mismo

6. Me siento bien cuándo creo que manejo los problemas lo mejor que puedo

7. Cuando tengo un problema o situación difícil, me tomo el tiempo necesario para comprender la situación y sus posibles soluciones

8. Siento que no puedo manejar los problemas o situaciones difíciles

9. Actúo con lentitud cuando tengo un problema o situación difícil

10. Cuando tengo un problema o situación difícil, intento orientar mis sentimientos y emociones para enfocarlos de forma provechosa

11. Cuando tengo un problema o situación difícil, me siento atento y activo durante el día

12 Cuando tengo un problema o situación difícil, intento que mis sentimientos no influyan para ver lo que sucede

13. Cuando tengo un problema o situación difícil, estoy pendiente de cualquier cosa relacionada con la situación

14. Tiendo a reaccionar de manera exagerada cuando tengo un problema o situación difícil

15. Cuando tengo un problema o situación difícil, tiendo a bloquearme y confundirme, al menos por un tiempo

16. Cuando tengo un problema o situación difícil, siento que es complicado hablar del problema

17. Me desempeño bien manejando problemas complicados

18. Cuando tengo un problema o situación difícil, busco todos los medios posibles para enfrentar la situación

19. Con el fin de salir de un problema o situación difícil puedo hacer cambios drásticos en mi vida

20. Mi capacidad para actuar mejora cuando me encuentro en situaciones estresantes

21. Puedo relacionar los problemas o situaciones difíciles con mis experiencias pasadas y con mis planes futuros

22. Tiendo a sentirme culpable por cualquier dificultad que tenga

23. Creo que mis experiencias pasadas no son útiles para resolver las situaciones difíciles o problemas

24. Utilizo las soluciones que le han funcionado a otros para resolver mis situaciones difíciles o problemas

25. Veo las situaciones difíciles o problemas como una oportunidad o un reto

26. Cuando tengo un problema o situación difícil, propongo diferentes soluciones para resolver la situación, aunque estas parezcan difíciles de alcanzar

27. Cuando tengo un problema o situación difícil, experimento cambios en el funcionamiento de mi cuerpo

28. Cuando tengo un problema o situación difícil, me hago cargo rápidamente y manejo las dificultades en la medida que van apareciendo

29. Cuando tengo un problema o situación difícil, intento aclarar cualquier tipo de duda relacionada con la situación antes de actuar

30. Cuando tengo un problema o situación difícil adopto rápidamente nuevas habilidades para resolverlo

31. Cuando tengo un problema o situación difícil, me rindo fácilmente

32. Cuando tengo un problema o situación difícil, desarrollo un plan que tiene una serie de acciones o pasos para

32. enfrentar la situación

33. Cuando tengo un problema o situación difícil, tengo muchas expectativas acerca de la manera cómo se resolverá la situación 
pitalarias y con el consentimiento informado por escrito de cada participante.

\section{RESULTADOS}

\section{Características sociodemográficas}

En total se encuestaron 404 cuidadores que dieron su consentimiento para participar en el estudio (tamaño de la muestra mínimo prestablecido, 400 participantes). Se incluyeron 124 de la CUB y 280 del HPTU, todas eran mujeres, con una mediana de edad de 30 años (RIC 2537); el grado de escolaridad más frecuente fue técnico y tecnólogo, 119 de ellas (29.5\%). De los cuidadoras con información disponible sobre estrato socioeconómico, 334 (84,6\%) pertenecían a los estratos uno, dos y tres. La mayoría profesaba alguna religión 146 (37.0\%) (Tabla 2).

Tabla 2. Características sociodemográficas de 404 cuidadoras de niños hospitalizados en dos instituciones de Medellín, Colombia.

\begin{tabular}{lc}
\hline Características & $\mathbf{n}(\%)$ \\
\hline Edad (años), n: 400, mediana (RIC ${ }^{+}$) & $30(25-37)$ \\
\hline Grado de escolaridad & \\
Primaria incompleta & $19(4.7)$ \\
Primaria completa & $18(4.5)$ \\
Bachillerato incompleto & $44(10.9)$ \\
Bachillerato completo & $106(26.2)$ \\
Técnico-tecnólogo & $119(29.5)$ \\
Universitario & $74(18.3)$ \\
Posgrado & $24(5.9)$ \\
\hline Estado civil, n: 403 & \\
Unión Libre & $160(39.7)$ \\
Casada & $130(32.3)$ \\
Soltera & $92(22.8)$ \\
Separada & $17(4.2)$ \\
Viuda & $4(1.0)$ \\
\hline Estrato socioeconómico, n: $\mathbf{3 9 5}$ & \\
1 & $79(20.0)$ \\
2 & $146(37.0)$ \\
3 & $109(27.6)$ \\
4 & $37(9.4)$ \\
5 & $19(4.8)$ \\
6 & $5(1.3)$ \\
\hline Pertenece a alguna religión, n: 403 & $374(92.8)$ \\
\hline Religión, n:374 & \\
Católica & \\
Cristiana & \\
Otras* & \\
\hline & \\
\hline
\end{tabular}

*Adventista, testigos de Jehová. ${ }^{\dagger} \mathrm{RIC}$ : rango intercuartílico.
En cuanto a las características de los niños hospitalizados, la mediana de edad fue de 24 meses (RIC 2-72). Los principales diagnósticos durante la hospitalización fueron las afecciones respiratorias, $113 / 398$ (28.4\%), seguido de las neurológicas, 46 (11.6\%\%) (Tabla 3).

Tabla 3. Características de los niños hospitalizados en dos instituciones de Medellín, Colombia.

\begin{tabular}{lc}
\hline Características & $\mathbf{n}(\%)$ \\
\hline Edad (meses), mediana (RIQ) & $24(2-72)$ \\
\hline Diagnósticos principales, n: 398 & $113(28.4)$ \\
\hline Afecciones respiratorias & $46(11.6)$ \\
\hline Afecciones neurológicas & $36(9.0)$ \\
\hline Afecciones del tracto urinario & $32(8.0)$ \\
\hline Enfermedades en estudio & $30(7.5)$ \\
\hline Enfermedades oncológicas & $25(6.3)$ \\
\hline Afecciones del tracto digestivo & $20(5.0)$ \\
\hline Infecciones sistémicas & $18(4.5)$ \\
\hline Alteraciones del metabolismo & $17(4.3)$ \\
\hline Patologías quirúrgicas & $11(2.8)$ \\
\hline Afecciones dermatológicas & $10(2.5)$ \\
\hline Afecciones osteomusculares & $8(2.0)$ \\
\hline Afecciones perinatales & $8(2.0)$ \\
\hline Bajo peso al nacer y prematurez & $7(1.8)$ \\
\hline Enfermedades hematológicas & $6(1.5)$ \\
\hline Enfermedades autoinmunes & $5(1.3)$ \\
\hline Afecciones cromosómicas & $5(1.3)$ \\
\hline Afecciones cardiacas & $1(0.3)$ \\
\hline
\end{tabular}

Se observó que gran parte de las cuidadoras principales eran las madres biológicas, 349 (86.4\%). El número de horas invertidas en el cuidado del niño fue en su mayoría de 24 horas, para 332 cuidadoras (82.4\%); casi todas contaron con apoyo, $283(70.2 \%)$ y la persona que les ayudó con más frecuencia fue el padre del niño 164 (58.0\%) (Tabla 4). 
Tabla 4. Características de las cuidadoras de 404 niños hospitalizados en dos instituciones de cuarto nivel de Medellín, Colombia.

\begin{tabular}{lc}
\hline Características & n (\%) \\
\hline Tipo de cuidador & \\
Madre biológica & $349(86.4)$ \\
Madre sustituta & $15(3.7)$ \\
Madre adoptante & $1(0.2)$ \\
Abuela & $1(0.2)$ \\
No reporta & $38(9.4)$ \\
\hline Horas invertidas en el cuidado, n: 403 & \\
$\leq 12$ & $40(9.9)$ \\
13-23 & $31(7.7)$ \\
24 & $332(82.4)$ \\
\hline ¿Cuenta con apoyo para el cuidado?, & $283(70.2)$ \\
n: 403 & \\
\hline ¿Quién la apoya?, n: 283 & $164(58.0)$ \\
Padre & $81(28.6)$ \\
Abuelos & $63(22.3)$ \\
\hline Otros familiares* &
\end{tabular}

*Otros familiares: primos, tíos, hermanos.

\section{Grado de afrontamiento y adaptación de las} cuidadoras

En la Tabla 5 se muestran los grados de afrontamiento y adaptación de las cuidadoras y una descripción según las variables sociodemográficas más frecuentes. La mediana del puntaje global fue 56 puntos, con RIC de 50-60, lo que ubica a la mayoría en un grado bajo-medio de afrontamiento; solo nueve (2.2\%) cuidadoras tenía un nivel de afrontamiento alto. Además, se observa que el grado bajo de afrontamiento es el más común, independientemente del estrato socioeconómico, estado civil y la religión.

\section{DISCUSIÓN}

Hay pocos estudios relacionados con el afrontamiento y adaptación de los cuidadores principales de niños hospitalizados o en el ámbito ambulatorio, menos aún en el caso de trabajos que utilizan la escala validada de Callista Roy. Las cuidadoras que participaron en este estudio eran mujeres (madres y abuelas), situación similar a la reportada en los trabajos de Campiño et $\mathrm{al}^{5}$, donde el 100\% fueron mujeres; Suárez y Monroy ${ }^{6}$, donde el

Tabla 5. Grado de afrontamiento y adaptación de las cuidadoras, según variables sociodemográficas, evaluado mediante la EsCAPS abreviada

\begin{tabular}{|c|c|c|c|c|}
\hline \multirow{2}{*}{ Variables } & \multicolumn{4}{|c|}{ Puntaje en la EsCAPS abreviada } \\
\hline & $\begin{array}{c}\text { Total, } \\
\text { mediana (RIC) }\end{array}$ & $\begin{array}{c}\text { Bajo (0-56) } \\
\text { n (\%) }\end{array}$ & $\begin{array}{c}\text { Medio (57-70) } \\
\text { n (\%) }\end{array}$ & $\begin{array}{c}\text { Alto (71-99) } \\
\mathbf{n}(\%)\end{array}$ \\
\hline Población total de cuidadoras & $56(50-60)$ & $219(54.2)$ & $176(43.6)$ & $9(2.2)$ \\
\hline \multicolumn{5}{|l|}{ Tipo de cuidador } \\
\hline Biológica (n: 349) & $56(51-60)$ & $185(53.0)$ & $157(45.0)$ & $7(2.0)$ \\
\hline Sustituta (n: 15) & $60(52-67)$ & $6(40.0)$ & $8(53.3)$ & $1(6.7)$ \\
\hline \multicolumn{5}{|l|}{ Estado civil } \\
\hline Unión Libre (n: 160) & $55(50-59)$ & $97(60.6)$ & $57(35.6)$ & $6(3.8)$ \\
\hline Casada (n: 130) & $56(50-60)$ & $66(50.8)$ & $62(47.7)$ & $2(1.5)$ \\
\hline Soltera (n: 92) & $56(52-62)$ & $47(51.1)$ & $44(47.8)$ & $1(1.1)$ \\
\hline \multicolumn{5}{|l|}{ Grado de escolaridad } \\
\hline Bachillerato completo (n: 106) & $55(50-60)$ & $60(56.6)$ & $44(41.5)$ & $2(1.9)$ \\
\hline Técnica-tecnólogo (n: 119) & $54(50-60)$ & $72(60.5)$ & $45(37.8)$ & $2(1.7)$ \\
\hline Universitario (n: 74) & $57(52-62)$ & $34(45.9)$ & $38(51.4)$ & $2(2.7)$ \\
\hline \multicolumn{5}{|l|}{ Estrato socioeconómico } \\
\hline $1(\mathrm{n}: 79)$ & $56(51-61)$ & $41(51.9)$ & $36(45.6)$ & $2(2.5)$ \\
\hline 2 (n: 146) & $55(50-60)$ & $85(58.2)$ & $56(38.4)$ & $5(3.4)$ \\
\hline 3 (n: 109) & $55(50-60)$ & $60(55.0)$ & $48(44.0)$ & $1(0.9)$ \\
\hline 4 (n: 37) & $56(52-60)$ & $20(54.1)$ & $17(45.9)$ & 0 \\
\hline \multicolumn{5}{|l|}{ Pertenece a alguna religión } \\
\hline Sí (n: 374) & $56(50-60)$ & $202(54.0)$ & $163(43.6)$ & $9(2.4)$ \\
\hline No (n: 29) & $55(51-62)$ & $16(55.2)$ & $13(44.8)$ & 0 \\
\hline
\end{tabular}


69\% lo era; Enríquez et al $1^{7}, 78.9 \%$; Ochoa et al ${ }^{8}, 79,3 \%$ y Tobo et $\mathrm{al}^{9}, 76 \%$.

En el contexto social y cultural, que se identificó en esta pesquisa, las mujeres son cuidadoras, encargadas de la crianza de los hijos y las labores del cuidado de estos. Cabe resaltar que la mujer aparte de ocuparse del cuidado del paciente tiene otras tareas, como el cuidado de otros hijos, el hogar y otras diversas laborales. En la tradición cultural latina, la maternidad es un componente importante de la identidad femenina ${ }^{10}$, y el proceso de asumir el rol de cuidado está muy relacionado con este género $^{12}$. Esta multiplicidad de tareas genera sobrecarga y contribuye al bajo grado de afrontamiento y adaptación.

La mayoría de las cuidadoras eran adultas jóvenes, lo que es similar a lo reportado por Tobo et $\mathrm{al}^{9}$, quienes encontraron que el 46\% era menor de 26 años. Suárez y Monroy ${ }^{6}$ refieren que el $47.8 \%$ de los padres estaba en adultez temprana y Campiña et $\mathrm{al}^{5}$ reportan una media de 39 años. Por su parte, Enríquez et $\mathrm{al}^{7}$ refieren que el $63 \%$ de los cuidadores está en el rango de 21 a 40 años. Ser madre joven de hijos hospitalizados es arduo, debido a la falta de experiencia y habilidades para desempeñar tareas complejas de cuidado, que necesitan esfuerzo, que requieren tomar decisiones difíciles sobre la situación del hijo ${ }^{13}$.

Aguiñaga et $\mathrm{al}^{14}$ estudiaron el estrés de los padres con hijos hospitalizados y refieren que el 51\% estaba en edades entre 15 y 24 años; el $25 \%$ presentó estrés moderado y el $35 \%$ reportó estrés severo. De la misma manera, el estudio de Stremler et al ${ }^{15}$ indica que el 24\% de los padres tenía ansiedad grave. El estudio de Konuk Şener D, Karaca $\mathrm{A}^{16}$ refiere que la hospitalización de un hijo es una experiencia estresante para los padres, y que la comunicación abierta y terapéutica entre padres y enfermeras contribuye a mejorar la tensión, tanto en padres como en los niños. En estos casos, el apoyo que reciben las madres es de gran ayuda para paliar las dificultades con las que se encuentran y para desarrollar las funciones que han de realizar.

En este estudio, el estado civil más común fue la unión libre. Esto también lo reportaron Ochoa et $\mathrm{a}^{8}$, quienes indican que el $48.3 \%$ de las cuidadoras era conviviente, al igual que en el estudio de Suárez y $\mathrm{Monroy}^{6}$, donde el $43.6 \%$ de las participantes vivía en unión libre. En el análisis de Tobo et $\mathrm{al}^{9}$, el $76 \%$ tenía una relación estable. El apoyo de la pareja es muy útil en momentos difíciles como la hospitalización de un hijo. Melguizo et al argumentan que los padres pueden participar más activamente en el cuidado, lo que reduciría el estrés que produce la protección de un hijo enfermo y afirman que la participación de la pareja en el cuidado es el único factor que aumenta la satisfacción vital ${ }^{13}$.

$\mathrm{El}$ estrato socioeconómico en el presente estudio fue principalmente bajo, al revisar la literatura, de acuerdo con los estudios de Campiño et al ${ }^{5}$ (estrato dos, 43.5\%), Suárez y Monroy $^{6}$ (estrato dos 30.9\%) y Tobo et al ${ }^{9}$ (estratos dos y tres, 80\%). Las malas condiciones económicas de las madres dificultan el desplazamiento, la alimentación e, inclusive, el hospedaje, en el caso de madres que no residen en la ciudad. Estas condiciones afectan la capacidad de afrontamiento.

En cuanto al grado de escolaridad, en el presente trabajo el más frecuente fue el técnico-tecnológico, a diferencia del estudio de Campiño et $\mathrm{al}^{5}$ (bachillerato incompleto en un 34.8\%). Suárez y Monroy ${ }^{6}$ dicen que el $50.7 \%$ tenía educación básica; Tobo et $\mathrm{al}^{9}$ manifestaron que el $36.7 \%$ había realizado el bachillerato completo. El mayor nivel educativo de las madres podría ayudar a afrontar la situación difícil de tener un hijo hospitalizado, ya que se correlaciona con mejor comprensión de la situación; sin embargo, ese no fue el caso en este estudio, donde las madres tuvieron un grado bajo de afrontamiento. Es necesario estudiar poblaciones con grado universitario para evaluar mejor la relación entre formación y afrontamiento.

Por otra parte, la mayoría de los cuidadores profesaba una religión y la principal fue la católica, muy similar a lo evidenciado por Campiño et $\mathrm{al}^{5}$, quienes manifiestan que la mayoría de las cuidadoras profesan la religión católica (73.9\%), seguida de la cristiana (17.4\%). La investigación de Crowther y Hall ${ }^{17}$ refiere que la espiritualidad y las creencias religiosas proporcionan apoyo en la toma de decisiones durante situaciones críticas. Vargas ${ }^{18}$ afirma que la religión es un componente importante de la espiritualidad, que influye sobre otras dimensiones de la persona, como su salud, bienestar y calidad de vida. Carreño y Chaparro ${ }^{1}$ expresan que, desde la dimensión espiritual, el cuidador entiende que su vida, planes y expectativas se transformaron y encuentra estrategias para el afrontamiento de la experiencia.

La mayoría de las cuidadoras invierte la mayor parte de su tiempo en el cuidado del niño hospitalizado, lo que sumado a otras obligaciones que deben resolver, produce estrés y dificultades para un afrontamiento eficaz; en este sentido, Xolyanetzin et $\mathrm{al}^{10}$ señalan que el $74 \%$ de los cuidadores primarios de niños con cáncer tiene sobrecarga, si se considera que el $70 \%$ cuida a sus niños por más de nueve horas diarias y realiza diversas actividades que requieren tiempo y dedicación. Se ha demostrado que la calidad de vida de los pacientes se relaciona con el estado emocional de sus cuidadores ${ }^{15}$; por tanto, los profesionales de enfermería pueden ofrecer estrategias a los cuidadores para tornar menos traumática la hospitalización. Las estrategias incluyen posibilitar expresar la experiencia y ampliar el conocimiento de los padres ${ }^{19}$. La comunicación y las relaciones abiertas y terapéuticas, entre padres y enfermeras, mejoran la calidad de la atención brindada a los niños y sus familias ${ }^{16}$. 
Esta investigación no buscó explorar relaciones entre las variables sociodemográficas y el nivel de afrontamiento, pero se observó que independiente del estado civil, la escolaridad, el estrato socioeconómico y la religión, las madres biológicas tuvieron un grado bajo de afrontamiento y adaptación. Campiño et $\mathrm{al}^{5}$ reportan hallazgos distintos en cuidadores de niños con cáncer, encontraron que el $56.5 \%$ de los cuidadores tuvo buen grado de afrontamiento.

En el estudio de Suárez y Monroy ${ }^{6}$ se hizo un análisis comparativo con algunas variables sociodemográficas, como la edad y el nivel de escolaridad de los padres, pero no hallaron diferencias en el proceso de afrontamiento y adaptación en el postoperatorio de niños con cardiopatía congénita.

En el presente estudio la mayoría de los cuidadores respondieron que tuvieron el apoyo de otras personas, sin embargo, el afrontamiento de los cuidadores punteó bajo. Suárez y Monroy ${ }^{6}$ identificaron que, los padres con hijos en postoperatorio de cardiopatías tuvieron un afrontamiento global alto, y argumentan que los cuidadores se beneficiaron de un apoyo adicional, porque asistieron a un programa social denominado "Regale una vida". El estudio sobre aspectos psicológicos en padres de niños hospitalizados en estado crítico ${ }^{15}$ encontró que el apoyo es protector de la salud mental.

Las patologías complejas de los niños y su manejo en un contexto hospitalario de alta complejidad, en este estudio, afectan la capacidad de afrontamiento y adaptación de las madres, quizás por el estrés al que están sometidas. En este sentido, Enríquez et al ${ }^{7}$ estudiaron cuidadores de niños con trastorno por déficit de atención con hiperactividad y encontraron un grado medio de afrontamiento en el 52.6\%; sin embargo, esos resultados no son comparables con los nuestros, debido a las diferencias en las características de las poblaciones.

El afrontamiento y la adaptación, según el modelo de Callista Roy, considera que las personas hallan en su entorno diferentes estímulos que desencadenan el proceso de adaptación, lo que da lugar a una respuesta eficaz o ineficaz ${ }^{20}$, en este estudio, el afrontamiento y la adaptación de las madres con hijos hospitalizados fue bajo, dado que las estrategias de afrontamiento de los cuidadores no incluyeron respuestas eficaces para mantener la adaptación.

Por otro lado, la escala utilizada implica algunas limitaciones para este estudio: algunos cuidadores manifestaron que la encuesta era larga, con preguntas complejas y repetidas, a pesar de haber usado la versión abreviada. También fue necesario ayudar a algunas madres para comprender el significado de varias preguntas. No fue posible reportar los resultados de acuerdo con los factores descritos para la versión original de la escala, ya que el análisis de componentes principales de la versión en español usada no es satisfactorio ${ }^{4}$.

En conclusión, esta investigación proporciona información que permite fortalecer los planes de cuidado para los cuidadores de niños hospitalizados, dado que el grupo de enfermeras que participó en el presente estudio pudo reconocer el impacto negativo de la hospitalización de los hijos sobre las madres. A partir de la información obtenida, se pueden implementar intervenciones para ayudar al afrontamiento y favorecer la adaptación de las madres, que impacten positivamente sobre su bienestar y sobre la salud de sus hijos.

Durante el curso de esta investigación se logró la motivación en los profesionales para diseñar un programa multidisciplinario de intervención, para promover estrategias de afrontamiento que disminuya la carga de los cuidadores.

\section{Declaración de conflictos de intereses}

Los autores declaran no tener conflictos de intereses.

\section{REFERENCIAS}

1. Gutiérrez C. Escala de medición del proceso de afrontamiento y adaptación de Callista Roy: una propuesta metodológica para su interpretación. Hallazgos. 2009;6: 209-13.

2. Flórez L, Villalobos M, Gallego P, Gómez B, González Y, Gutierrez M, et al. Análisis de concepto del modelo de adaptación de Callista Roy. Aquichan 2002;2:19-23.

3. Gutiérrez C, Veloza MM, Moreno ME, Durán MM, López C, Crespo O. Validez y confiabilidad de la versión en español del instrumento "Escala de medición del proceso de afrontamiento y adaptación" de Callista Roy. Aquichán 2007;7:54-63.

4. Sarmiento P, Botero J, Carvajal G. Validez de constructo de la Escala de Medición del Proceso de Afrontamiento y Adaptación de Roy, versión modificada en español. Index Enferm 2013;22:233-6.

5. Campiño S, Duque P. Afrontamiento y adaptación de cuidadores de niños y niñas con cáncer. Universidad y Salud 2016;18: 302-11.

6. Suárez C, Monroy A. Afrontamiento y adaptación de los padres durante el postoperatorio de cardiopatía congénita. Index Enferm 2012;21:145-9. 
7. Enríquez C, Alba LC, Corzo L, Caballero D, Rojas D. Adaptación, afrontamiento y calidad de vida en cuidadores de infanto-juveniles con trastorno por déficit de atención con hiperactividad. Acta Med Cent 2018;12:57-63.

8. Ochoa K, Villaizan M, Tam-Phun M, Gutiérrez P. Relación entre el funcionamiento familiar y la capacidad de afrontamiento y adaptación de la familia con un niño con problema neurológico crónico del Servicio de Neuropediatría del Instituto Nacional de Salud del Niño. Rev Enferm Herediana 2016;9:101-7.

9. Tobo N, Betancur P, de la Cruz C. Estímulos, afrontamiento y adaptación en padres de recién nacidos hospitalizados en unidades de cuidado intensivo neonatal. Investigación en Enfermería: Imagen y Desarrollo 2017;19:161-75.

10. Montero X, Jurado S, Méndez J. Variables que predicen la aparición de sobrecarga en cuidadores primarios informales de niños con cáncer. Psicooncología 2015;12: 67-6.

11. Enríquez C, Alba L, Pérez M, Alonso DD. Programa psicoeducativo enfermero para cuidadores de infantojuveniles con trastorno por déficit de atención con hiperactividad. Rev Cubana Enferm. 2018;34:[aprox. 0 p.].

12. Carreño-Moreno SP, Chaparro-Díaz L. Calidad de vida de los cuidadores de personas con enfermedad crónica. Aquichan 2016;16:447-61.

13. Melguizo-Garín A, Martos-Méndez MJ, Hombrados-Mendieta I. Influencia del apoyo social sobre el estrés y la satisfacción vital en padres de niños con cáncer desde una perspectiva multidimensional. Psicooncología 2019;16:25-42.

14. Aguiñaga-Zamarripa $\mathrm{ML}$, Reynaga-Ornelas $\mathrm{L}$, Beltrán-Torres A. Estrés percibido por los padres del neonato en estado crítico durante el proceso de hospitalización. Rev Enferm IMSS 2016;24:27-35.

15. Stremler R, Haddad S, Pullenayegum E, Parshuram C. Psychological outcomes in parents of critically ill hospitalized children. J Pediatr Nurs 2017;34:36-43.

16. Konuk D, Karaca A. Mutual expectations of mothers of hospitalized children and pediatric nurses who provided care: Qualitative study. J Pediatr Nurs 2017;34:e22-e28.

17. Crowther S, Hall J. Espiritualidad y cuidado espiritual en y alrededor del parto. Mujer y Nacimiento 2015;28:173-8.

18. Vargas LM. Validez y confiabilidad de la versión en español de la Escala de Evaluación de la Espiritualidad y el Cuidado Espiritual. Rev Colomb Enferm 2015;11:34-44.

19. Bazzan JS, Milbrath VM, Gabatz RI, Cordeiro FR, Freitag L, Schwartz E. O processo de adaptação familiar à hospitalização infantil em Unidade de Terapia Intensiva. Rev Esc Enferm 2020;54:e03614.

20. Naranjo Y. Puntos de encuentro entre las teorías de Roy y Orem en la atención a ancianos con úlcera neuropática. Rev Cubana Enferm 2019;35(2):[aprox. 0 p.]. 